\title{
Las organizaciones geoculturales latinas en la mundialización: entre convergencia y concurrencia
}

\author{
AyMERIC Durez
}

\begin{abstract}
Artículo recibido: 28 de febrero de 2014
Artículo aprobado: 28 de marzo de 2014

Doi: dx.doi.org/10.12804/desafios26.02.2014.05

Para citar este artículo: Durez, A. (2014). Las organizaciones geoculturales latinas en la mundialización: entre convergencia y concurrencia. Desafíos, 26 (2), 125-152. doi: dx.doi. org/10.12804/desafios26.02.2014.05
\end{abstract}

\section{Resumen}

En la escena internacional, todos los Estados actúan dentro de marcos colectivos que se centran en la defensa y la promoción de intereses comunes. Ulteriormente a la descolonización, nuevas uniones entre Estados, fundadas desde el compartir de una lengua comun, florecieron. En particular, entre los hispanobablantes, los francófonos y los lusófonos, la toma de conciencia sobre los intereses comunes generó la creación de organizaciones intergubernamentales. Después de haber hecho un recuento bistórico de dichas organizaciones, desde un análisis comparado, este texto analiza su nuevo rol en el marco de la mundialización y se interroga en particular acerca de su cohabitación con las organizaciones regionales.

Palabras clave: Organización de Estados Americanos, Organización Internacional de Francofonía, Comunidad de países de Lengua Portuguesa, organizaciones geoculturales, lenguas.

\footnotetext{
* Investigador en el Institut de la Francophonie et de la Mondialisation (Iframond), Universidad de Jean Moulin Lyon 3, Francia. Correo electrónico: aymeric.durez@univ-lyon3.fr
} 


\title{
Geocultural Organizations in Globalization: Between Convergence and Divergence
}

\begin{abstract}
In the international arena, all states act in collective entities that focus on the defense and promotion of common interests. Following the process of decolonization, new unions between states emerged in the context of sharing a common language. Particularly, Spanish-speaking, French-speaking and Portuguese-speaking people, taking consciousness of their mutual interests, created intergovernmental organizations. After a historical review of those organizations from a comparative perspective, this article analyses their role in the context of globalization and inquires about their co-existence with regional organizations.

Keywords: Organization of Ibero-American States, Organisation Internationale de la Francophonie, Community of Portuguese Language Countries, geocultural organizations, language.
\end{abstract}

\section{As organizações geo-culturais latinas na mundialização: Entre convergência e concorrência}

\begin{abstract}
Resumo
Na cena internacional, todos os Estados atuam dentro de marcos coletivos que se centram sobre a defensa e a promoção de interesses comuns. Ulteriormente à descolonização, novas uniões entre Estados fundadas sobre o compartilhamento de uma lingua comum, floresceram. É em particular entre os hispanoparlantes, os francófonos e os lusófonos, que a toma de consciência sobre os interesses comuns gerou a criação de organizações intergovernamentais. Depois de ter feito uma recontagem histórica de ditas organizaçoes, desde uma análise comparada, este texto analisa seu novo rol no marco da mundialização e se interroga em particular sobre sua coabitação com as organizações regionais.

Palavras chave: Organização dos Estados Ibero-americanos, Organização Internacional da Francofonia, Comunidade dos Países de Lingua Portuguesa, organizações geo-culturais, línguas.
\end{abstract}




\section{Introducción}

Si bien la lengua es una herramienta de definición de identidad para los individuos y los pueblos, ella desempeña también un papel preponderante en la formación de los procesos de legitimización de los Estados. Dentro de las fronteras, la lengua es una herramienta unificadora, a la vez que puede constituir un instrumento de poder en las relaciones exteriores (Chauprade, 2007). Desde el siglo XIX, el inglés se impuso como la lengua predominante a escala mundial. En primer lugar, mediante la potencia imperial inglesa, y después por el acceso de los Estados Unidos al rango de primera potencia mundial. Para este último país, el inglés es un mecanismo de dominación política, económica y, sobre todo, cultural, pues constituye el medio de transmisión del modo de vida estadounidense.

Ante la dominación del inglés, numerosos Estados han reaccionado legislando para defender su lengua en el interior de sus fronteras y han adoptado políticas de promoción lingüística en el extranjero. Los institutos Confucio, Dante, Cervantes, Pouchkine y la Alianza Francesa están inscritos dentro de esta estrategia. No obstante, en la "guerra de las lenguas", que se juega a escala mundial, ciertos países también han escogido reagruparse al seno de organizaciones geoculturales fundadas desde el compartir un idioma común (Calvet, 2005). Mientras que es más común que los Estados se asocien bajo un fundamento geográfico a partir de la ecuación: proximidad geográfica = prioridad política, la institucionalización de comunidades lingüísticas ofrece un nuevo modelo de organizaciones intergubernamentales (Charillon, 2013).

A la fecha, las organizaciones mejor estructuradas se encuentran en el seno de los espacios hispanoparlantes, francófonos y lusófonos. ${ }^{1}$ Estas organizaciones geolingüísticas nacieron de la voluntad compartida

\footnotetext{
1 Si el Commonwealth inspiró la constitución de espacios geoculturales latinos, en razón de su naturaleza histórica demarcada por su proximidad a la corona británica y la ausencia de una política centrada en la lengua, es un caso particular. De la misma forma, si la liga árabe puede ser considerada como la organización arabofónica, es en principio la unidad religiosa lo que está en la base de la organización.
} 
entre las antiguas potencias coloniales españolas, francesas y portuguesas y los nuevos países independientes; así como de conservar una relación especial organizando una cooperación institucionalizada, donde se aplica el principio de igualdad entre sus miembros. Mientras que la mayor parte de países que han obtenido la soberanía internacional han tomado conciencia del interés de adoptar la lengua de la antigua potencia colonial como la lengua oficial, este patrimonio común justifica el mantenimiento de una acción conjunta y la defensa de intereses comunes en la escena internacional.

Durante largo tiempo, el paso de la existencia de intereses comunes a la construcción efectiva de proyectos orgánicos fue complicado para la primacía de las uniones ideológicas dentro del contexto de la Guerra Fría y bajo el espectro del neocolonialismo. Sobre este último punto, la emergencia de una o varias potencias concurrentes de la antigua potencia colonial parece haber favorecido la institucionalización de los vínculos geoculturales. En razón de la existencia de los diferentes "Estados piloto", la francofonía, la lusofonía y la hispanofonía se diferencian de la turcofonía y la rusofonía, donde la preponderancia de Turquía y Rusia constituye un obstáculo para la ejecución de uniones geoculturales (Raptopoulos, 2007; Massart-Piérard Françoise, 2007).

Si la hispanofonía, la francofonía y la lusofonía tienen la vocación de organizar una política de cooperación cultural y comparten la voluntad de luchar a favor de la multipolaridad lingüística, la convergencia entre los tres espacios lingüísticos está amenazada por contradicciones internas y rivalidades entre las tres organizaciones. Además, a pesar del ensanche de los sectores de intervención de dichas organizaciones, varios obstáculos se imponen para que estas logren hacerse admitir como legítimas y creíbles en el multilateralismo mundial.

\section{Organizaciones nacidas de la descolonización}

Debido al movimiento independentista en el siglo XIX, la primera organización geolingüística fundada en el ámbito intergubernamental estuvo constituida por España y sus antiguas colonias en América Latina. Tras negar la independencia a sus colonias, España intentó 
conservar cierta influencia en América latina. Así, poco antes del colapso final de su imperio de ultramar, en 1898, dio un primer paso con la creación de una Unión Iberoamericana, en 1885 (Pereira Castañares, 1992). Pero después de la segunda guerra mundial nació una iniciativa intergubernamental. En la reunión de 1954, en Quito, para el Segundo Congreso Iberoamericano de Educación, los países hispanoparlantes decidieron transformar la Oficina de Educación Iberoamericana (OEI), fundada 1949, en una organización intergubernamental que reúne a los Estados soberanos.

Teniendo como objeto construir una mayor solidaridad entre los pueblos iberoamericanos, la OEI ha permitido establecer una defensa común de la lengua española y la cooperación en los sectores culturales, educativos y científicos. ${ }^{2}$ Sin embargo, la voluntad de la dictadura franquista de utilizar los lazos iberoamericanos no permitió una cooperación consecuente. Entonces, después de 1976, y sobre todo a partir de 1982, dichas iniciativas florecen.

Dentro del espacio francófono, es el movimiento de descolonización en África que entrañó la creación de proyectos comunes. Los jefes de Estado africanos: Léopold Sédar Senghor, Habib Bourguiba y Hamani Diori fueron los principales promotores de la idea de la francofonía, tras el descalabro de la comunidad francesa en 1958, como el último baluarte del imperio francés (Turpin, 2010); no obstante, la voluntad de los jefes de Estado se vio obstruida por la oposición de un cierto número de países africanos, en particular Argelia, y debido a la reserva

2 La OEI tiene veinticinco Estados miembro: Andorra, Argentina, Bolivia, Chile, Colombia, Costa Rica, Cuba, Ecuador, El Salvador, España, Guatemala, Guinea Ecuatorial, Honduras, México, Nicaragua, Panamá, Paraguay, Perú, Puerto Rico, República Dominicana, Uruguay, Venezuela, Brasil y Portugal. Brasil se adhirió a la OEI por primera vez en 1980 y nuevamente lo hizo en 1999, después de decidir que no sería solamente un miembro observador en 1983. Portugal también se unió a la organización en 1999. La presencia de los dos países lusófonos evidencia la intención de la OEI de promover de la misma manera la lengua española y la portuguesa. Sin embargo, la organización sigue estando marcada por los vínculos hispanohablantes que se demuestra por la ausencia de los países africanos de lengua oficial portuguesa (PALOP). Según un miembro de la OEI, a quien se interrogó, Brasil y Portugal ejercen una cooperación entre ambos separada del seno de la OEI y además no existe una política que apoye la lengua portuguesa en los países hispanohablantes ni viceversa. 
francesa. El general de Gaulle, poco inclinado por las organizaciones multilaterales, privilegió las políticas diferenciadas en el interior del espacio francófono. Prosiguió con una política de cooperación bilateral con los países africanos, y un apoyo en las comunidades francesas del exterior, como Quebec, así como con la defensa del fait français, dentro de cada una de las regiones del mundo. A pesar de ello, una organización intergubernamental modesta, la Agencia de Cooperación Cultural y Técnica (ACCT), terminó fundándose el 20 de marzo de 1970, en Niamey.

El nacimiento de la lusofonía está igualmente vinculado con la emancipación de las colonias portuguesas en África alrededor de 1975. No obstante, la constitución de una organización intergubernamental fue imposible durante mucho tiempo, debido a dos principales obstáculos: en primer lugar, a causa de la querella lingüística entre Portugal y Brasil, y en segundo lugar, debido a la adhesión de Angola y Mozambique a la ideología marxista. Ambos obstáculos fueron superados con el fin de la Guerra Fría y con la firma de un Acuerdo de Ortografía en Lisboa, el 12 de octubre de 1990, entre la Academia de Ciencias de Lisboa y la Academia Brasileña de Letras.

La Comunidad de Países de Lengua Portuguesa (CPLP), primera organización intergubernamental de lusofonía, nació durante el curso de la cumbre de los jefes de Estado en Lisboa, el 17 de Julio de 1996³ (Santana, 2007). El artículo tercero, del acuerdo suscrito en la cumbre de 1996, estipula que la CPLP se dedicará a "promover un vasto proyecto político cuyo fundamento es la lengua portuguesa, como vínculo histórico y patrimonial común de los siete miembros".

\section{La constitución de las organizaciones geoculturales como punto de equilibrio de la construcción europea}

Para Portugal, la creación de la CPLP es un medio que permite reafirmar su vocación de ultramar, dentro de un contexto marcado por

\footnotetext{
3 La CPLP tiene ocho miembros: Portugal, Brasil, Angola, Cabo Verde, Guinea Bissau, Mozambique, Santo Tomás y Príncipe y Timor Oriental, que se integró a la CPLP tras su independencia, en 2002. También tiene tres observadores: Guinea Ecuatorial, Senegal y Mauricio.
} 
la europeización de su política exterior desde 1976 (Teixeira, 2003; MacQueen, 2003). Sin embargo, la aceleración de la construcción europea al final de los años ochenta tuvo repercusiones en cada una de las tres organizaciones geolingüísticas. En una obra publicada en enero de 1986 titulada: Reflexiones sobre la politica exterior francesa, el presidente François Mitterrand, quien gobernó Francia de 1981 hasta 1995, reafirmó su apego a la construcción de Europa, al mismo tiempo que expresó su voluntad de defender la lengua francesa y de poner en acción un reagrupamiento de países francófonos, un mes antes de la celebración de la primera cumbre francófona de Versalles (17-19 febrero de 1986):

Desde el principio de siglo — escribió entonces- la renuncia de Francia a los atributos de su soberanía no tiene límite. Es en Bruselas donde se han formulado los objetivos de la política agrícola, en La Haya en donde se juzgan los procesos internacionales en los que nuestros intereses están en juego, y en Luxemburgo en donde se juzgan los conflictos internos de la comunidad. [...] Me regocijo de que en nuestro planeta reducido, se agranda el campo contractual. No obstante, existen ciertos aspectos, para nada insignificantes, y es en donde reivindico nuestro patrimonio, el cual ha sido infringido, y el que debe ser reconquistado y devuelto a Francia. Dentro de este patrimonio destacó en primer lugar nuestra lengua [...] Nadie escucha a un pueblo que ha perdido sus palabras. A través de la escuela, por los medios audiovisuales, la formación de intérpretes, la reagrupación activa de las naciones francófonas, con la ayuda de las asociaciones educativas y ante todo, por el orgullo y el amor propio que aporta al mundo nuestra lengua, es que podremos revertir esa tendencia (Mitterrand, 1986, p. 14).

El paralelo efectuado entre la dinámica de integración europea y la voluntad de preservar ciertas prerrogativas nacionales, entre las cuales se encuentra la posibilidad de participar en una organización francófona, traduce adecuadamente la voluntad de encontrar un cierto equilibrio entre política europea y política de grand large (Arnaud, Guillou y Salon, 2005). Al mismo tiempo, para los dirigentes africanos, la institucionalización del proyecto de la francofonía garantiza el mantenimiento de 
un vínculo privilegiado con Francia, así como la posibilidad de hacer escuchar sus voces sobre la orientación de la construcción europea.

La reunión de la primera cumbre francófona en Versalles no solamente estuvo marcada por la creación de proyectos de cooperación, sino por un examen de la situación política mundial, que desencadenó la adopción de resoluciones comunitarias. El viraje político de la francofonía enseguida fue consagrado en 1997 con la adopción de la Carta de Hanói, cuyo preámbulo proclama "la necesidad de darle a la francofonía su plena dimensión política”. La creación de una Secretaría General responde a esta necesidad, así como la transformación de la ACCT en una agencia de la francofonía que fue rápidamente bautizada con el nombre de Organización Internacional de la Francofonía (OIF).

En cuanto a la OIE, es la perspectiva del ingreso de España en la Unión Europea, en 1986, la que entrañó una nueva reflexión sobre el provenir del proyecto iberoamericano. Durante una conferencia eurocentroamericana, en San José de Costa Rica, el 29 de septiembre de 1984, el ministro de Asuntos Exteriores de España, Fernando Morán, dijo: "No hay contradicción entre nuestra vocación europea y nuestra vocación americana, sino que es posible que España aporte una contribución sustancial a este nuevo dialogo" (citado en Del Arenal, 1984, s. p.).

La organización cambió de dimensión durante la reunión extraordinaria del Congreso de la OEI en Bogotá, en mayo de 1985. Más allá de la adopción de una nueva denominación - la Oficina de Educación Iberoamericana convertida en la Organización de Estados Americanos, OEI-, el Congreso se destacó por la adopción de nuevos estatutos, que muestran una mayor ambición de la organización. La transformación de la OEI en una clase de "Unesco hispanoparlante" permitió a España reafirmar los vínculos culturales e históricos con los países latinoamericanos; mientras que su ingreso en la Unión Europea consagró la integración de su economía dentro del mercado único europeo (Drago, 1985). 
Al igual que la francofonía, los países iberoamericanos decidieron crear conferencias entre sus jefes de Estado y la primera cumbre tuvo lugar en 1991, en Guadalajara, México. Si bien es cierto que la OEI ha suscitado el nacimiento de este tipo de cumbres, las dos entidades continúan funcionando de forma independiente, mientras que las conferencias de los jefes de Estado de la francofonía y de la lusofonía constituyen las instancias supremas de la OIF y de la CPLP. ${ }^{4}$ Sin relevo para imponer su visión en las relaciones entre los países hispanoparlantes, la Cumbre ante todo desempeña un papel como foro de discusión. Empero, en el 2003, durante la Tercera Cumbre Iberoamericana, celebrada en Bolivia, se creó la Secretaría General Iberoamericana (SEGIB), con el objeto de dar mayor visibilidad a la organización en la arena internacional, asegurar una mayor continuidad entre las cumbres y organizar una mejor concertación con las distintas organizaciones iberoamericanas. ${ }^{5}$

El nacimiento de la SEGIB dio un matiz político a las cumbres, al incitar a los países miembro a adoptar una acción común en las organizaciones internacionales y a buscar soluciones a los interrogantes del desarrollo y la inmigración. Finalmente, en 2004, la creación del Consejo de Organismos Iberoamericanos permitió implementar un mecanismo de coordinación y diálogo entre las diferentes organizaciones iberoamericanas. Esta nueva organización permite una mejor cohesión y refuerza la importancia de las cumbres, ya que está presidida por el secretario general de la SEGIB. ${ }^{6}$

\section{El acercamiento de las organizaciones respecto a la promoción de la diversidad cultural}

Las tres organizaciones "fónicas" se han aproximado en sus estructuras y objetivos en los últimos años. La existencia de las secretarías

\footnotetext{
4 Véase el artículo 3 de la Carta d'Antanarivo, del 23 de noviembre de 2005, para la francofonía y el artículo 8 de los estatutos de Lisboa del 17 de julio de 1996 para la CPLP.

5 Todos los miembros de la OEI son también miembros de las cumbres, a excepción de Guinea Ecuatorial y de Puerto Rico, que tienen únicamente el estatus de miembros observadores, al igual que Filipinas, desde 1999, estatus al cual Francia, Italia, Países Bajos, Bélgica y Mónaco accedieron en 2010.

${ }_{6}$ Desde abril de 2014 la costarricense Rebeca Grynspan.
} 
generales garantiza a cada una un perfil más alto en la escena internacional. La OEI-SEGIB, la CPLP y la OIF se acercaron también a la Organización de las Naciones Unidas con la firma de alianzas y acuerdos (Julia, 2008; Tavares y Bras, 2011). Las organizaciones geoculturales, con su fundamento lingüístico, buscan ubicarse como actores de relaciones internacionales junto a las organizaciones internacionales de carácter universal y agrupaciones regionales.

La utilidad y la eficacia de las tres organizaciones han salido a la luz a través de la lucha común por el voto de la Convención sobre la "protección y promoción de la diversidad de las expresiones culturales" en la Unesco, adoptada el 20 de octubre de 2005 por 48 votos, cuatro abstenciones y dos votos en contra. El presente convenio marca el advenimiento de una ley cultural internacional (Farchy y Tardif, 2011; Rodríguez Barba, 2008). En su preámbulo, proclama explícitamente la especificidad de los bienes y servicios culturales, explicando que no deben ser considerados mercancías sujetas a las leyes del libre comercio. Como resultado de ello, se dice que los Estados tienen el derecho de desarrollar políticas de apoyo a las industrias culturales, que pueden tomar la forma de cuotas de difusión y medios financieros para la producción y distribución de dichos productos y servicios.

Para la hispanofonía, la francofonía y lusofonía, la adopción de la Convención de la Unesco es la culminación de una lucha que comenzó en la década de los noventa, como parte de las negociaciones de la Ronda de Uruguay (1984-1994), para contrarrestar la voluntad de los Estados Unidos de integrar los bienes y servicios culturales a las negociaciones del Acuerdo General sobre Aranceles Aduaneros y Comercio sobre la liberalización de los servicios mundiales de comercio. En 1993, la francofonía se pronunció contra esta liberalización, durante una reunión cumbre en la Isla de Mauricio (16-18 de octubre de 1993), y solicitó que las actividades culturales fueran excluidas del ámbito de aplicación de tal Acuerdo en aras de una "excepción cultural”. El 15 de abril de 1994, el Acta Final de la Ronda de Uruguay en Marrakech otorgó un plazo de diez años para la aplicación del principio de excepción cultural. Tras esta decisión, se sostuvo un primer contacto entre las organizaciones geoculturales latinas durante una conferencia 
en París, el 17 y 18 de marzo de 1995, titulada "Alternativas para la uniformización".

En 2001, en preparación para la reanudación de las negociaciones sobre la liberalización, la OEI, la OIF, la CPLP y la Unión Latina, en ocasión de un simposio titulado Tres Espacios Lingüísticos se Enfrentan a los Retos de la Globalización, decidieron unir sus fuerzas para hacer reconocer el principio de la diversidad cultural. El cambio de la "excepción cultural" hacia la "diversidad cultural" traduce el paso de una estrategia defensiva para las industrias de los países del norte a una dinámica más igualitaria en favor de todas las expresiones culturales. Una nueva estructura común: Tres Espacios Lingüísticos (3EL) se creó. En los años siguientes, los secretarios generales de los 3EL organizaron reuniones a intervalos periódicos, siempre entremezcladas con reuniones de expertos, para discutir las opciones legales para bloquear la liberalización de bienes y servicios culturales.

Reunidos en México del 2 al 4 de abril de 2003, con ocasión de un nuevo coloquio, los responsables de los 3 EL adoptaron una declaración que proclama: "la diversidad cultural y lingüística es un valor irrenunciable, en tanto es manifestación de la tendencia de los grupos humanos a sus desenvolvimiento en condiciones de variedad, y fuente esencial de la creatividad humana a la vez que un factor de dinamismo, originalidad e interacción de los pueblos" (Cooperación, diversidad y paz, 2003, p. 196). Al año siguiente, los secretarios generales de los $3 \mathrm{EL}$ se encontraron en la sede de la Unesco. Después de una reunión con los embajadores de los países miembro de las tres organizaciones geoculturales, ellos sostuvieron una conferencia de prensa común para dar su apoyo a la adopción de la Convención.

La adopción de la Convención de 2005 se puede considerar un verdadero éxito para las tres organizaciones que han sabido formar una coalición de intereses para que la Unesco legisle en favor de la diversidad cultural. Sin embargo, este éxito se ve desvalorado, por un lado, debido a que la Convención ha sido en gran medida evitada por los Estados Unidos a través de la firma de acuerdos bilaterales de libre comercio y, por otro, debido a que la Unesco tiene medios limitados 
para ejecutar la mencionada Convención. En efecto, a diferencia de la Organización Mundial del Comercio, ella no cuenta con un órgano de solución de conflictos.

Por otra parte, cabe señalar que si la cuestión de la protección de las industrias culturales ha sido un punto importante de acuerdo entre los países de las tres organizaciones geoculturales, en su mayoría, tienen que hacer frente a la diferencia entre los intereses de los Estados nacionales miembro. La búsqueda del más pequeño común denominador dentro de cada organización y la competencia entre las regiones lingüísticas hacen muy aleatoria la adopción de una política común en el ámbito internacional.

\section{El papel particular de las antiguas metrópolis}

En los juegos de poder que animan la hispanofonía, la francofonía y la lusofonía, las metrópolis antiguas tienen un lugar especial. Debido a su antiguo estatus de potencia colonial y "cuna de la lengua", España, Francia y Portugal ocupan un lugar sustancial en el seno de la hispanofonía, francofonía y lusofonía. La presencia de las sedes de la OEI, OIF y la CPLP en Madrid, París y Lisboa ilustra esta importancia particular, como el hecho de que los tres países europeos han sido siempre los principales contribuyentes financieros dentro de su respectiva organización.

La membresía a una organización geocultural permite a España, Francia y Portugal mantener una capacidad de alcance global, a pesar de la prioridad que se da a la construcción europea. Sin embargo, estos países también deben hacer frente a los intereses de otros Estados y no pueden permitirse un dominio real. Por el contrario, saben que al tratar de imponer sus criterios de forma ostentosa, serán acusados de neocolonialismo por parte de sus socios. Las acusaciones de querer volver a la época de la hispanidad, de la francité y del lusotropicalismo penden como una espada de Damocles encima de los tres países europeos. Dentro de las organizaciones nacidas, tras romper el vínculo colonial, España, Francia y Portugal deben tratar de convencer a sus socios sobre sus puntos de vista y buscar compromisos. 
En la CPLP, Portugal busca imponer la lengua portuguesa, pese a lo reducido de su territorio y a continuar con su atávica política de proyección en ultramar. Sin embargo, Portugal aparece como un enano demográfico y económico frente a Brasil, quien desempeñó un papel importante a favor de la creación de la CPLP en la década de los ochenta, bajo el liderazgo de los presidentes Itamar Franco (1992-1995) y Fernando Henrique Cardoso (1995-2003). Dentro de la organización, Brasil ha desarrollado estrechas relaciones con los países africanos de lengua oficial portuguesa (PALOP), en el contexto de una estrategia global dirigida a aumentar su presencia en África. Debido a su peso demográfico y económico, Brasil se disputa el liderazgo de la organización con Portugal, y a pesar del acuerdo ortográfico de 1990, la competencia lingüística entre los dos países no se ha detenido. Después de haber aprobado una ley para la defensa y promoción de la lengua portuguesa, frente a la invasión del inglés (Ley 1976/1999), Brasil fundó en 2006 el Instituto Machado, encargado de la difusión de la cultura brasileña en el mundo, que compite con el Instituto Internacional del Portugués fundado en 2001 y respaldado por Portugal (Santana Ferra, 2007).

Dentro de la OEI y la Cumbre Iberoamericana, España ha aparecido siempre en primer lugar; pero la crisis económica y social en el país ha debilitado significativamente su posición, mientras que los países de América Latina han estado experimentando un crecimiento sostenido. Sobre dicho punto, la reciente Cumbre Iberoamericana de Cádiz (16-17 de noviembre de 2012) se caracterizó por una inversión de la relación de poder entre España y sus antiguas colonias. En respuesta al llamado del rey Juan Carlos para una mayor solidaridad en el espacio iberoamericano, varios líderes de América del Sur, entre ellos la presidenta de Brasil, Dilma Rousseff, y el jefe de Estado colombiano, Juan Manuel Santos, se ofrecieron a ayudar a España a salir de la crisis. En la Cumbre se estableció un comité de sabios para considerar un cambio de estructura en la Secretaría General y una reflexión sobre el papel de la Cumbre y su periodicidad. Por un lado, está establecido que la Cumbre no será anual, sino bienal, alternada con las cumbres que reúnen los países de la Unión Europea y la Comunidad de Latinoamericanos y Caribeños. Por otra parte, el Comité podría pronun- 
ciarse sobre un reequilibrio financiero, ya que España financia el 60\% de los gastos de funcionamiento de la Secretaría General (Gualdoni, González y de Sandoval, 2012).

En el seno de la francofonía, Francia continúa ocupando un lugar central (Agger, 1996; Tavares y Bras, 2005). A través de su red diplomática mundial y el puesto permanente en el Consejo de Seguridad de las Naciones Unidas, ella tiene ventajas decisivas para hacerse portavoz de los países francófonos. Las intervenciones que realiza en Malí y en la República Centroafricana reflejan una respuesta tanto del potencial de su herramienta militar como su influencia en el continente africano. Empero, en el seno de la francofonía, Francia debe compartir el mando con Canadá (Thérien, 1993; Lefèvre, 2010). Desde las primeras cumbres, los canadienses han sabido explotar los miedos de Francia de ser acusada de neocolonialismo por imponer sus prioridades ${ }^{7} \mathrm{y}$, por ello, Canadá ha insistido constantemente para que la francofonía debata cuestiones de política internacional bajo el modelo de las cumbres del Commonwealth. Más recientemente, Canadá estimuló la adopción de la Declaración de San Bonifacio, sobre la prevención de conflictos y la seguridad humana. La influencia de Francia en la francofonía está también limitada debido al gran número de Estados miembro de la organización. Con 77 Estados (57 miembros y 20 observadores), la OIF es la organización más heterogénea, y debe lidiar con países de intereses muy diferentes.

\section{La francofonía: ¿una organización geocultural piloto?}

Si bien es cierto que los 3 EL se componen de Estados muy diversos desde el punto de vista geográfico, cultural, económico y social, la francofonía ofrece el mejor ejemplo de diversidad en su interior (Abou, Crouzatier, Ruxandra y Soppelsa, 2010). En el seno de la OIF ocupan escaños tanto miembros de la Unión Europea como miembros de la Asociación de Naciones del Sudeste Asiático, de la Unión Africana, y desde hace poco también del Mercado Común del Sur (Mercosur). ${ }^{8}$

Archivos de Erick Arnoult 5AG4/EA 36, dossier 2.

8 Tras la entrada de Uruguay en 2012 como miembro observador. 
La diferencia en cuanto al grado de desarrollo de los Estados miembro también se hace importante, porque se encuentran países que poseen un índice de desarrollo humano muy alto (superior a 0,9), muy débil $(0,5 / 0,6)$ y extremadamente débil (menos de 0,5$)$.

Desde el punto de vista religioso, existe también una gran diversidad, ya que la francofonía está en países de tradición cristiana, budista, animista e islámica (diecinueve miembros de la OIF pertenecen a la conferencia islámica). Por tanto, en el plano lingüístico, el estatus del francés depende de cada miembro y sus características particulares.

Si bien es cierto que la francofonía se estableció desde el principio con países que no tienen el francés como lengua oficial, eran países, en su mayoría, con un pasado colonial compartido con Francia o Bélgica, y todos ellos tenían una fuerte relación con la lengua francesa. El fin de la Guerra Fría llevó a la francofonía a abrirse. Desde 1991, Rumania y Bulgaria se adhirieron a la organización, junto con Vietnam. Con estos ingresos comenzó un largo periodo de expansión de la francofonía: mientras que 41 países asistieron a la cumbre en Dakar, en 1989, fueron 57 los participantes en la Cumbre de Kinshasa, en 2012. Se hizo una amplia apertura, especialmente para los países del este de Europa, con la Cumbre de Moncton, en 1999.?

La francofonía es la única organización geocultural que tomó dicho camino. Por el contrario, en el caso de la OEI-SEGIB y de la CPLP, el estatus de miembro pleno sigue siendo el privilegio de los países de lengua oficial hispana y portuguesa. El artículo 6.1 del estatuto de la CPLP establece como criterio principal, para pertenecer a la organización, tener como lengua oficial el portugués. Si, por el contrario, en la OEI-SEGIB no aparece un criterio tal es porque el campo de la organización está delimitado por el espacio geolingüístico iberoamericano. Por otra parte, para la francofonía el texto sobre las modalidades y estatus de adhesión a la organización, adoptado en la cumbre de

\footnotetext{
9 Durante el desarrollo de la cumbre, Albania y Macedonia se convirtieron en miembros asociados a diferencia de Lituania, Eslovenia y República Checa, que obtuvieron un estatus de observadores dentro de la organización.
} 
Beyrouth, en 2002, hace prevalecer como criterio de adhesión la voluntad de promover la lengua francesa por parte de las autoridades del país candidato, sobre el estatus que se le da al francés en dicho país y su difusión cuantitativa dentro.

El ensanche de la organización, al largo plazo, incluyendo los países que tienen una socialización muy baja con el francés, ha estado presente como un requisito para aumentar el peso de la organización en la escena internacional. Si bien esta evolución ha llevado a la OIF a conectarse con la mayoría de las organizaciones internacionales y a ser reconocido como actor en la resolución de conflictos, se ha visto también revestida de una cierta crisis de identidad. Ciertas críticas nacieron debido a la doble evolución de expansión de la francofonía y la adopción de nuevas prioridades políticas. En 2002, el embajador de Francia, Bernard Dorin, actor histórico en la construcción francófona, subrayó que "desde el momento en que algunos países, que no tienen mucho que ver con la francofonía, se aceptan, se diluye considerablemente la naturaleza misma de la organización" (Comeau Fournier, 2002) $\cdot{ }^{10}$ En respuesta a las críticas sobre una expansión imprudente y una brecha cada vez mayor entre la composición geopolítica y la realidad lingüística de la OIF, se inició una ofensiva en favor de la socialización del francés durante la Cumbre de Quebec en 2008 (Phan, Guillou y Durez, 2012). Sin embargo, la entrada controversial de Qatar en la OIF, en la última Cumbre en Kinshasa, decisión tomada por los jefes de Estados, en contra de la opinión de la organización, plantea nuevas preguntas sobre el sentido de la francofonía. Un reporte reciente de la Comisión de Asuntos Extranjeros del parlamento francés recomendó, igualmente, la creación de un círculo restringido de países francófonos para obtener una mayor coherencia (Rochebloine y Amirshahi, 2014).

La ampliación de la organización y el paso de una francofonía poscolonial a una francofonía pos-Guerra Fría han estado acompañados de la adopción de nuevos objetivos. La promoción de la democracia, los

\footnotetext{
10 Bernard Dorin fue uno de los principales militantes de la causa de Quebec y de la francofonía en los años sesenta. Igualmente, fue el primer director del Departamento de los Asuntos Francófonos en el Ministerio de Relaciones Exteriores desde la fecha de su creación, en 1974.
} 
derechos humanos y el Estado de derecho se convirtieron en los temas centrales en la organización. Estos asuntos comenzaron a imponerse durante la Cumbre de Chaillot (19-21 de noviembre de 1991), donde los jefes de Estado de la francofonía adoptaron una resolución dirigida "al proceso de democratización, la consolidación de las instituciones democráticas y a desarrollar programas en esta dirección". Consecutivamente, la adopción de la Carta de Hanói hizo del "desarrollo de la democracia, la prevención de conflictos y el apoyo del gobierno de derecho y los derechos humanos", uno de los principales objetivos de la organización (artículo 1).

La Carta también creó un puesto de Secretaría General de la Francofonía encargada de ser "el portavoz político y representante oficial de la francofonía”. El puesto fue confiado al egipcio Boutros Boutros Ghali, quien contribuyó considerablemente a hacer de la francofonía un actor del multilateralismo en la escena internacional. Como secretario general de las Naciones Unidas (1992-1996), Boutros Ghali favoreció el acercamiento entre la francofonía y las Naciones Unidas dentro de su agenda por la paz, bajo la cual buscaba crear una gran colaboración entre esta entidad y el conjunto de organizaciones regionales (Julia, 2008). Elegido como primer secretario general de la francofonía, en 1997, durante la Cumbre de Hanói, Boutros Ghali acentuó la proximidad entre las dos organizaciones, así como la OIF fue admitida como parte de las organizaciones con estatus de observador dentro de las Naciones Unidas. ${ }^{11}$

La adopción de la Declaración de Bamako, que proclama que "la democracia y la francofonía son indisociables" y que "no puede haber una profundización del proyecto francófono sin un progreso constante hacia la democracia y su realización en los hechos" (artículo 3.1),

\footnotetext{
11 Las diez organizaciones "regionales" que disponen a la vez de un estatus de observador en las Naciones Unidas y una representación permanente en ellas son: la Unión Africana, el Comité Jurídico Consultativo para los Países de Asia y África, la Comunidad Caribe, el Sistema de Integración Centroamericano, el Commonwealth, el Consejo de Cooperación de los Estados Árabes del Golfo, la Unión Europea, la Organización Internacional de la Francofonía, la Liga de los Estados Árabes y la Organización de la Cooperación Islámica. La CPLP y la SEGIB solamente cuentan con el estatus de observadores, mas no con el de miembros permanentes.
} 
confirmó la transformación histórica de la organización francófona, haciendo de la adopción de un sistema democrático un criterio preponderante de pertenencia a la organización sobre el criterio lingüístico. También se confirmó el compromiso de la francofonía en desempeñar un papel más importante en la resolución de las crisis en los países de habla francesa pues, desde la Declaración de Bamako, proporciona un mecanismo de reacción en tres etapas: "Mantenerse constantemente informada de la situación la democracia, los derechos humanos y las libertades en el mundo francófono" (artículo 5.1). En caso de crisis de la democracia o la violación grave de los derechos del hombre, los órganos de la francofonía se apropiarán del tema, "para tomar las iniciativas para prevenir la agravación y contribuir para la creación de una reglamentación" (artículo 5.2) de los problemas. Por último, "en caso de fracaso de la democracia o la seria violación de los derechos humanos", la Declaración de Bamako exige del secretario general una reacción. Este debe convocar de inmediato al Consejo Permanente de la Francofonía, que puede tomar una serie de medidas restrictivas y decidir suspender al miembro (artículo 5.3). ${ }^{12}$

\section{Nuevos actores en la resolución de los conflictos}

Desde la adopción de la Declaración de Bamako, la francofonía ha adquirido un potencial innegable en materia de resolución de crisis y de acompañamiento democrático (Bagayoko y Ramel, 2013; Vettovaglia, 2010; Maila, 2010). La OIF participa regularmente en grupos internacionales de contacto al lado de los actores tradicionales (organizaciones internacionales y regionales, potencias locales e internacionales). Sin embargo, la CPLP busca, igualmente, ejercer un rol dentro del ámbito político-diplomático. Durante una conferencia de ministros de la defensa de Praia, en Capo Verde, el 25 de mayo de 1998, la CPLP fue dotada con un programa de cooperación militar. Al mismo tiempo, la organización ejecutó una misión de buenos oficios en Guinea Bissau. Además, desde 2000, la CPLP realiza ejercicios militares conjuntos cada año a través de un programa denominado FELINO. La nueva

\footnotetext{
12 En febrero de 2014, Guinea-Bisau, Madagascar y República Centroafricana están suspendidos de las instancias de la francofonía.
} 
prioridad dada a los temas de defensa fue consagrada en 2001, con la adopción de una revisión de estatutos en 1996. Finalmente, cada año, los ministros lusófonos de defensa se reúnen con el fin de reforzar las sinergias entre las diferentes fuerzas armadas y de apoyar el refuerzo de las capacidades de los Estados miembro. En 2006, los miembros de la conferencia adoptaron un "protocolo de cooperación en materia de defensa entre los países de lengua portuguesa". Al largo plazo, la CPLP busca poder desplegar una fuerza común bajo la autorización de las Naciones Unidas (Tavares y Bras, 2005).

Las acciones de la OIF y de la CPLP se explican por la multiplicación de conflictos intergubernamentales en África subsahariana desde principios de los años noventa. A causa de su débil presencia en África y de su homogeneidad geográfica, la OEI-SEGIB no está en capacidad de aportar una plusvalía en materia de resolución de conflictos respecto a las organizaciones regionales como Mercosur o la Organización de Estados Americanos. Si la Carta de la OEI reivindica el compromiso de los países miembro con la "construcción de la paz", así como con "la defensa de los derechos humanos", la cooperación cultural y la educación permanecen como los únicos vectores de sus objetivos.

Sobre este punto, la OIF y la CPLP presentan muchas más semejanzas. Las dos organizaciones se impusieron como actores de resolución de conflictos en África, y bajo el capítulo vi de la Carta de las Naciones Unidas, ellas ejecutan una acción en materia de mediación. Igualmente, disponen de programas en cuanto al refuerzo de las capacidades institucionales de sus Estados miembro y, por otra parte, ellas han adquirido cierto conocimiento en materia de observación electoral. Si las organizaciones geoculturales aparecen aún como actores de segundo plano, a causa de sus medios económicos débiles y de su legitimidad limitada respecto a los demás actores, su rol podría vigorizarse. Mientras que el llamado en favor de un reglamento regional sobre las crisis africanas se traduce generalmente en meros deseos, las organizaciones geoculturales tienen la ventaja de ofrecer a la vez una cohesión cultural y lingüística con una complementariedad norte-sur. Así, las Naciones Unidas han empezado a asociar la francofonía con sus operaciones para el sostenimiento de la paz, en la medida en que la OIF constituye 
un relevo para permitir una participación más amplia y más eficaz de los países francófonos en sus OMP ${ }^{13}$ (Massie y Morin, 2011).

Si la OIF y la CPLP reivindican un rol más fuerte como organizaciones geoculturales en la resolución de crisis, las dos organizaciones no comparten necesariamente los mismos principios y métodos para ejecutar dicha acción.

La OIF dispone de una ventaja, en cuanto a la prevención de las crisis. Ella se apoya, a la vez, en su vasta red institucional, puesta a disposición de la delegación a los derechos humanos, a la democracia y a la paz, y en el prestigio de su secretario general, el antiguo presidente de Senegal, Abdou Diouf. Sin embargo, la acción multilateral de la francofonía en materia de defensa es aún prematura y la organización no prevé crear una fuerza operacional propia. Por otra parte, las crisis recientes de la primavera árabe y en África subsahariana, que dieron lugar a apreciaciones aleatorias de la declaración de Bamako por los Estados miembro de la OIF, han puesto bajo la luz pública la dificultad para la francofonía de fundar sus acciones sobre principios éticos (Colonomos, 2005; Charillon, 2007). Mientras que la Declaración de Bamako prevé una condena automática a "los golpes de Estado y toda otra toma del poder por medios violentos, las armas o cualquier otro medio ilegal" (artículo 3.5), los golpes de Estado en Níger (18 de febrero de 2010), en Egipto (4 de julio de 2013) y en la República Centroafricana (24 de marzo de 2013) fueron objeto de reacciones diferentes desde la ausencia de condena como en el caso de Egipto, hasta la condena y suspensión en el caso de la República Centroafricana. ${ }^{14}$

Contrariamente a la OIF, la CPLP no posee mecanismos de sanción o de suspensión para con sus miembros y la organización respeta rigurosamente el principio de no intervención en los asuntos de los Estados (artículo 5b). Esta política tiene la ventaja de asegurar la CPLP contra las derivas de las injerencias, mientras que el principio de responsabilidad de proteger, adoptado por la francofonía en la Declaración de

\footnotetext{
13 A/RES $/ 65 / 263$.

$14 \mathrm{CP} / \mathrm{SG} / \mathrm{JT} / 06 / 10 ; \mathrm{CP} / \mathrm{SG} / 25 / \mathrm{JT} / 13$ y $\mathrm{CP} / \mathrm{SG} / 13 / \mathrm{JT} / 13$.
} 
San Bonifacio, en 2006, ha sido deslegitimizado, debido a su instrumentalización durante la crisis en Libia.

Sin embargo, la CPLP sufre de una falta de legitimidad. En razón de su modestia y del compromiso limitado de Brasil, la organización no consigue crear vínculos sustanciales sobre la escena internacional. Así, la voluntad de poner a disposición de las Naciones Unidas una fuerza operacional de la CPLC se ve obstaculizada por la mala coordinación de las organizaciones regionales africanas (Tavares y Bras, 2005).

\section{Organizaciones concurrentes}

Las organizaciones geolingüísticas no solo se caracterizan por la influencia de las luchas internas. A pesar de la solidaridad mostrada entre la OEI, la OIF y la CPLP y la implementación de políticas comunes, estas organizaciones aparecen a veces en situaciones de competencia que, por lo general, se originan en la defensa y promoción de los diferentes idiomas (Bieger Merkli, 2010). Por lo tanto, aunque las organizaciones se comprometieron en la conferencia en México en 2003 en una lucha en conjunto para defender la presencia del español, el portugués y el francés en las organizaciones internacionales, cada organización se centra principalmente en aumentar la importancia de la lengua de la que es responsable, para así desafiar el monolingüismo inglés. Este fenómeno se refleja en la situación lingüística de la Unión Europea, donde el multilingüismo institucional que pone en posición de igualdad a las veinticuatro lenguas oficiales se traduce, en la práctica, en el reinado del inglés (Grin, 2005).

La cuestión de la pertenencia a las organizaciones geoculturales también causa una cierta competencia lingüística entre el español, el francés y el portugués. Si bien es cierto que las esferas de influencia parecen bien delimitadas entre hispanofonía y lusofonía, debido a la presencia de Portugal y Brasil en la OEI-SEGIB, y la falta de competencia en África, ${ }^{15}$ no ocurre lo mismo vis-á-vis con la francofonía, cuya

\footnotetext{
15 Mientras que ningún Estado lusófono pertenece a la OEI o a la SEGIB, Guinea Ecuatorial, miembro observador de la CPLP, fue rechazado por segunda vez consecutiva como miembro
} 
apertura política crea algunas tensiones, especialmente en el espacio de habla portuguesa. La CPLP frunce el ceño frente a la influencia de la lengua francesa en las islas de Cabo Verde y Guinea Bissau; ${ }^{16}$ mientras que también se enfrenta a la competencia del Commonwealth en Mozambique (Cahen, 1999).

La rivalidad en África entre la CPLP y la OIF no se detiene solo en el área lingüística; a veces, los resultados se reflejan en el campo político. Es particularmente el deseo de ambas organizaciones, de participar en la resolución de conflictos en África, lo que hace aparecer intereses opuestos. En Guinea Bissau, la francofonía y la lusofonía se oponen constantemente desde el golpe de Estado de 1998. La rivalidad no se ejerce directamente entre las dos organizaciones geoculturales, sino entre la organización lusófona y la Comunidad Económica de los Estados de África Occidental, donde diez hacen parte de la OIF; mientras que la CPLP está representada únicamente por Guinea Bissau y Cabo Verde. En 2006, la OIF no tomó parte en la constitución de un grupo internacional de contacto formado por varios Estados y diversas instituciones internacionales. ${ }^{17}$ Esta "retirada" de la OIF traduce una voluntad clara de no alimentar la rivalidad entre las organizaciones geoculturales, pero las divergencias de intereses permanecen (Massey, 2004).

La crisis que ha sacudido a Guinea Bissau, en 2012, tras un golpe de Estado militar contra el primer ministro Gomes Junior es un buen ejemplo. Mientras que la OIF ha respaldado a la Comunidad Económica de los Estados de África Occidental ${ }^{18}$ como actor en la resolución de la crisis, la CPLP, bajo la dirección de Angola y Portugal, criticó la

pleno de la organización durante la última Cumbre de Maputo (Mozambique), en razón de faltas contra la democracia, según el reporte oficial.

16 Siendo miembros de la CPLP, Guinea Bissau y Cabo verde son igualmente miembros de la francofonía desde 1979 y 1996, respectivamente.

17 Angola, Brasil, Cabo Verde, España, Gambia, Gana, Guinea, Nigeria, Níger, Portugal, Senegal, la CPLP, la Unión Africana, el Banco Mundial, las Naciones Unidas, la Unión Económica y Monetaria de África Occidental, el Fondo Monetario Internacional, la Comisión de la Unión Europea y la Comunidad Económica de los Estados de África Occidental.

$18 \mathrm{CP} / \mathrm{SG} / 07 / \mathrm{JT} / 12$. 
organización subregional y reclamó asumir un papel más activo en la resolución del conflicto. Apoyando el fortalecimiento del papel de la Organización Regional Africana, las Naciones Unidas reconocieron a la CPLP el derecho a participar más activamente en la resolución de la crisis. ${ }^{19}$ Sin embargo, la CPLP tuvo que renunciar al despliegue de una operación para el mantenimiento de la paz.

\section{Conclusión}

Desde la adopción de la Convención del 20 de octubre de 2005, la colaboración entre las tres regiones lingüísticas titubeó, y cada organización persiguió sus propios objetivos. Sin embargo, de cara al arrollador paso anglosajón y el deseo de proyección mundial de los Estados Unidos, incluyendo el lenguaje y la diplomacia cultural como activos de primera, los $3 \mathrm{EL}$ tienen un interés en unirse para desarrollar una política común y establecerse como polos apartados de la globalización multipolar. En 2012, un grupo de trabajo creado por el secretario general de la OIF, Abdou Diouf, trabajó el tema multilingüismo y francofonía e hizo una propuesta para adoptar una Convención de la Unesco sobre el multilingüismo inspirado en la Convención de 2005 sobre la diversidad cultural. Tal proyecto permitiría a las organizaciones geoculturales participar en las discusiones para definir una alianza a favor del multilingüismo en todas las organizaciones internacionales.

Mientras que las organizaciones geoculturales padecen de una falta de compromiso por parte de los Estados miembro, una reflexión se impone en cada una sobre los ámbitos de competencias (Farchy y Tardif, 2011). Tanto para la francofonía como para las otras organizaciones, la urgencia de identificar retos comunes y definir una estrategia sostenible se hace sentir. Las tres organizaciones geoculturales podrían actuar en conjunto para hacerse reconocer dentro de un estatus específico internacional. Desde este punto de vista, su asimilación, hecha por las Naciones Unidas, al mismo nivel de las organizaciones regionales bajo el capítulo VIII de la Carta de las Naciones Unidas no es satisfactorio (Tavares y Bras, 2005).

19 S/RES/2048 (2012) . 
Además, el futuro de las organizaciones geoculturales como uniones intergubernamentales sigue estando condicionado a su articulación con las organizaciones regionales tradicionales. Los países miembros de los 3 EL pertenecen a grupos regionales o continentales diferentes, a los cuales les asignan mayor prioridad. Si bien la Unión Europea tiene el modelo de integración más avanzado, los países latinoamericanos también privilegian sus organizaciones regionales. La Comunidad de Latinoamericanos y Caribeños, la Unión de Naciones Suramericanas, Mercosur, la Alianza Bolivariana para los Pueblos de Nuestra América, la Comunidad Andina de Naciones y la Alianza del Pacífico ayudan a construir la integración continental de manera multidimensional en los ámbitos regional y subregional (Dierckxsens, 2013).

En África, varias organizaciones subcontinentales, como la Comunidad Económica de Estados de África Occidental y la Comunidad Económica de los Estados de África Central cohabitan con la Unión Africana. Además de la solidaridad regional, las relaciones establecidas con las grandes potencias como Estados Unidos, China y Rusia también pueden ser factores de divisiones dentro de las organizaciones geoculturales.

La falta de coordinación hizo que cierto número de políticas previstas en materia de cooperación económica o de inmigración se enfrentaran a las prerrogativas de las organizaciones regionales, en particular a la Unión Europea. En el centro de esta organización, los vínculos geoculturales mantenidos por los diferentes miembros constituyen relevos naturales para la política exterior y de seguridad. La entrada de España y Portugal a la comunidad europea, en 1986, desde este punto de vista, suscitó al mismo tiempo el nacimiento de una verdadera cooperación entre el continente europeo y América Latina y una reactivación de los vínculos iberoamericanos (Sberro, 2003). Sin embargo, el prisma anglosajón de la Unión Europea y el desplazamiento del centro de gravedad al este de Europa no contribuyen a la creación de vínculos geoculturales. El fracaso de la reactivación de un proyecto de Unión por el Mediterráneo demostró que Bruselas se acomodaba mal a la adhesión de ciertos miembros de organizaciones distintas. Además, si bien es cierto que el debilitamiento de las iniciativas ibe- 
roamericanas, simbolizado por la ausencia de varios jefes de Estado latinoamericanos en la última cumbre de Panamá, el 19 de octubre de 2013, está ligado en parte a razones internas, también es un reflejo de la incapacidad de Europa de valorizar los vínculos geoculturales para reforzar su política internacional.

Para darles mayor valor a los vínculos geoculturales, los países europeos podrían adoptar una política más independiente frente a los Estados Unidos, a fin de aparecer como verdaderos actores de la multipolaridad. Desde este punto de vista, el regreso de Francia al mando integrado de la Organización del Tratado del Atlántico Norte, en 2009, y el anuncio de España, para octubre de 2011, del establecimiento de una cesión de la base naval de Rota, cerca de Cádiz para el protector de misiles de la Organización del Tratado del Atlántico Norte son mensajes negativos para los países emergentes, ya que ello ilustra la prioridad acordada al vínculo con los Estados Unidos en detrimento de una política de mediación norte-sur(Debray, 2013).

\section{Referencias}

Abou, S., Crouzatier, J.-M. y Soppelsa, J. (2010). Solidarité en (fF)rancophonie: réalité ou faux-semblant? París: Agence universitaire de la Francophonie.

Agence Intergouvernementale de la Francophonie (1989). Francophone et Acte unique européen. Documento procedente de las Actes du Colloque International de Franceville, 23-26 janvier 1989, Agence de Coopération Culturelle et Technique.

Agger, D. (1996). Francophonie in the 1990s: problems and opportunities. Clevedon: Multilingual Matters.

Arnaud, S., Guillou, M. y Salon, A. (2005). Les défis de la Francophonie, pour une mondialisation bumaniste. París: Alphares.

Badji, M., Crouzatier, J.-M., Ivan, R. y Soppelsa, J. (2010). Francophonie et solidarité. Cluj: AUF.

Bagayoko, N. y Ramel, F. (2013). Francophonie et profondeur stratégique. Etude de l'RRSEM (26). Recuperado de http:/ / www.defense.gouv.fr/irsem/ page-d-accueil/vient-de-paraitre/etude-de-l-irsem-n-26-2013-francophonie-et-profondeur-strategique 
Bieger-Merkli, C. (2010). La communauté des pays de langue portugaise: un espace de coopération interculturel de coopération basé sur la langue. París: L'Harmattan. Cahen, M. (1999). Un point de vue français, Défendre la langue portugaise en Afrique ?, La nationalisation du monde, Europe, Afrique, l'identité dans la démocratie. París: l'Harmattan.

Calvet, L. J. (2005). La guerre des langues et les politiques linguistiques. Pluriel: Hachette.

Charillon, F. (2007). L'éthique: le nouveau mot d'ordre de la politique étrangère française? Revue Internationale et Stratégique, 3 (67), 87-94.

Charillon, F. (2013). Déclinaisons stratégiques du territoire. Etudes de l'IRSEM (4).

Chauprade, A. (2007). Géopolitique, constantes et changements dans l'bistoire (3a. ed.). París: Ellipses.

Comeau, P. A. y Fournier, J. P. (2002). Le lobby du Québec à Paris: les précurseurs du général. Montréal: Amérique.

Cooperación, diversidady paz (2-4 de abril, 2003). Documento procedente dle II Coloquio de los Tres Espacios Lingüísticos. México. Recuperado de http://www.oei.es/pensariberoamerica/IITEL.PDF

Debray, R. (mars, 2013). La France doit quitter l'otan. Le Monde Diplomatique (708).

Del Arenal, C. (2011). Politica exterior de España y relaciones en América Latina: iberoamericanidad, europeización y atlantismo en la politica exterior española. Madrid: Siglo XXI-Fundación Carolina.

Dierckxsens, W. (marzo, 2013). La geopolítica de la integración latinoamericana. Le Monde Diplomatique (120).

Drago, T. (1985). Iberoamérica, una definición que compromete. El País, 11 de junio. Recuperado de http://elpais.com/diario/1985/06/11/ espana/487288803_850215.html

Farchy, J. y Tardif, J. (2011). Les enjeux de la mondialisation culturelle. París: Bord de L'eau.

Grin, F. (2005). L'enseignement des langues étrangères comme politique publique. Report to the Haut Conseil de l'évaluation de l'école, Ministère de l'éducation nationale, París.

Gualdoni, F., González, M. y De Sandoval, P. X. (2012). La Cumbre de Cádiz pone las bases para "refundar" la comunidad iberoamericana. El País, 17 de noviembre. 
Julia, M. (2008). L'évolution de la coopération entre l'ONU et les organisations régionales: l'exemple de l'Organisation internationale de la Francophonie. En M. Guillou y P. Trang (Dirs.), La Francophonie sous l'angle des théories des Relations internationales. París: Iframond.

Lefèvre, M. (2010). Le soutien américain à la Francophonie: enjeux africains, 1960 1970. París: Les Presses de la Fondation Nationale des Sciences Politiques.

MacQueen, N. (2003). A community of illusions?: Portugal, the CPLP and peacemaking in Guinée Bissau. International Peacekeeping, 10 (2), 1-26.

Maila, J. (2010). La notion de crise en Francophonie : entre dispositif normatif et traitement politique. La Revue Internationale des Mondes Francophones (2), 24.

Massart-Piérard, F. (2007). Espaces linguistiques comparés : trajectoires et processus transversaux. Revue Internationale de Politique Comparée, 14 (1), 165-192.

Massey, S. (2004). Scientia militaria. South African Journal of Military Studies, $32(1)$.

Massie, J. y Morin, D. (2011). Francophonie et opération de paix, vers une appropriation géoculturelle. Revue Études internationales, XLII (3).

Mitterrand, F. (1986). Réflexions sur la politique extérieure de la France : introduction aux à 25 discours, 1981-1985. París: s. e.

Pereira Castañares, J. C. (1992). España e Iberoamérica: un siglo de relaciones (18361936) (pp. 97-127). Madrid: Casa de Velázquez.

Phan, T. y Guillou, M. (2011). Francophonie et mondialisation (t. 1: Histoire et institutions des origines à nos jours). París: Belin.

Phan, T. ; Guillou, M. y Durez, A. (2012). Francophonie et mondialisation (t. 2: Les grandes dates de la construction de la Francophonie institutionnelle). París: Belin.

Raptopoulos, N. (2007). La famille des langues turques et le défi de création d'une communauté turcophone en Eurasie : le rôle assumé par Ankara. Revue Internationale de Politique Comparée, 14, 131-150.

Reis Torgal, L. (2005). De l'Empire atlantique eurafricain à la communauté des pays de langue portugaise, Réalité, mythe et utopie. Matériaux Pour l'Histoire de Notre Temps (77).

Rochebloine, F. y Amirshahi, P. (2014). La Francophonie : action culturelle, éducative et économique. París: Commission des Affaires étrangères. 
Rodríguez Barba, F. (2008). La Convención sobre la Protección y la Promoción de la Diversidad de las Expresiones Culturales de la Unesco y su impacto en las políticas culturales de México. Desafíos (19), 11-47.

Rojas Aravena, F. (2000). Las cumbres iberoamericanas: una mirada global. Caracas: Nueva Sociedad.

Santana Ferra, F. (2007). Un espace phonique lusophone à plusieurs voix?: Enjeux et jeux de pouvoir au sein de la Communauté des pays de langue portugaise (CPLP). Revue Internationale de Politique Comparée, 14 (1), 95-129.

Sberro, S. (2003). L'Espagne, atout de l'Amérique latine dans l'Union européenne. Revue Internationale et Stratégique, 1 (49), 91-99.

Tavares, R. y Bras, B. L. (2005). Speaking the language of security: the Commonwealth, the Francophonie and the CPLP in conflicto Management in Africa. Conflict, Security and Development, 11 (5), 607-636.

Teixeira, N. S. (2003). Between Africa and Europe: Portuguese Foreign Policy 1890-1986. In A. C. Pinto (Ed.), Contemporary Portugal. New York: SSM-Columbia University Press.

Thérien, J. P. (1993). CO-operation and conflict in la francophonie. International Journal, XLVIII, Summer.

Turpin, F. (2010). De Gaulle, Pompidou et l'Afrique (1958-1974): décoloniser et coopérer. París: Les Indes Savantes.

Vettovaglia, J.-P. (2010). Médiation et facilitation dans l'espace francophone: théorie et pratique. Prévention des crises et promotion de la paix (vol. I). Bruxelles: Bruylant.

Vettovaglia, J.-P. (2013). Déterminants des conflits et nowvelles formes de prévention (vol. III). Bruxelles: Bruylant. 